\title{
DEVELOPING A WEB SCHEDULING SYSTEM BASED ON XML MODELING
}

\author{
Leonilde Rocha Varela ${ }^{1}$, Joaquim Nunes Aparício ${ }^{2}$, and Sílvio Carmo Silva ${ }^{3}$ \\ ${ }^{I}$ University of Minho, School of Engineering, Dept. of Production and Systems \\ leonilde@dps.uminho.pt \\ ${ }^{2}$ New University of Lisbon, Faculty of Science and Technology, Dept. of Computer Science \\ jna@di.fct.unl.pt \\ ${ }^{3}$ University of Minho, School of Engineering, Dept. of Production and Systems \\ scarmo@dps.uminho.pt
}

\begin{abstract}
In manufacturing enterprises, it is important nowadays, as a competitive strategy, to explore and use software applications, now becoming available through the Internet and Intranets, for solving scheduling problems. This work gives a contribution for a better resolution process of scheduling problems, by means of web-based computation. An XML-based specification framework for scheduling is proposed. This specification provides a general model of scheduling problems and related tasks, and consequently a method of describing each particular problem and algorithm, by user information introduction. The main objective of this kind of specification is to make possible flexible communication among different scheduling applications, in a web-based scheduling decision support system.
\end{abstract}

\section{INTRODUCTION}

Production Scheduling is an important function strongly contributing to the competitiveness of industrial and service companies. Manufacturing Scheduling may be defined as the activity of allocating tasks to production resources, during a certain period of time.

An effective and efficient resolution of a scheduling problem begins with the identification of suitable scheduling algorithms. When there are some alternative methods and algorithms to solve the problem it is important to make the evaluation of solutions based on the alternatives available and in accordance with the specified criteria or objectives to be reached. We should be able to solve a problem, through the execution of one or more scheduling algorithms and, choose the best solution provided by them. These algorithms can be local or remotely available through the web. Thus, this work intends to be a contribution for a better resolution process of scheduling problems, by means of web-based computation.

It is proposed an XML-based specification framework for manufacturing scheduling concepts modeling. This specification provides a general model of scheduling problems and related tasks, and consequently a method of describing each particular problem and algorithm. This requires identification of such 
problems, which can be classified by a set of factors, which, in turn, enable specifying a clear and objective problem categorization structure to which real problem instances belong. The existence of a great variety of scheduling problems motivates the utilization of a systematic notation for problems representation that serves as a basis for its classification (Varela, 1999). The nomenclature includes the manufacturing system identification, definition of the performance measure and some constraints that characterize the problem.

According to the notation used for representing scheduling problems, the XML language is used as a specification language for data modeling and processing. The main objective of this kind of specification is to make possible flexible communication among different scheduling applications, in a web-based scheduling decision support system. The specification also contributes to the improvement of the scheduling processes, by allowing an easier selection of several alternative algorithms or methods available for problem solving, as well as an easier maintenance of the knowledge base itself.

This paper is organized as follows. The next section describes the nature of scheduling problems and presents a nomenclature for problem characterization. Some algorithm references to common scheduling problem classes are also presented. Sections 3 and 4 describe the web-based scheduling system main functionalities. Section 4 shows a sample XML specification of scheduling problem classes and other related modeling aspects. The last section presents some concluding remarks and additional discussion points related to the future developments of this work.

\section{SCHEDULING PROBLEMS DESCRIPTION}

Scheduling problems belong to a much broader class of combinatorial optimization problems, which may either be easy or hard (NP-hard problems) to solve. Detailed information about complexity of scheduling can be found in (Blazewics, 1996), (Brucker, 1995), (Jordan, 1996) and (Pinedo, 1995). In the presence of NP-hard problems we may try to relax some constraints imposed on the original problem and then solve the relaxed problem. The solution of the latter may be a good approximation to the solution of the original one and only polynomial time algorithms are likely to be acceptable to solve complex problems. Fortunately, not all NP-hard problems are equally hard from a practical perspective. Some NP-hard problems can be solved pseudo-polynomially using approximation algorithms that generally provide only feasible solutions, which although normally sub-optimal are within a fixed percentage of the optimum. Examples of this kind of methods are the nowadays widely used local (or neighborhood) search techniques, such as Genetic Algorithms (GA), Simulated Annealing (SA), and Tabu Search (TS), which are also known as extended neighborhood search techniques. All these approaches tend to provide good results in the available time to take decisions, reasons why, in this work, we intend to incorporate them in the web scheduling system we are developing.

Good schedules strongly contribute to the increase companies' success. This is achieved, among other ways, through deadlines satisfaction for the accepted orders, low flow times, few ongoing jobs in the system, low stock levels, high resource 
utilization and low production costs. All these objectives can be better satisfied through the execution of the most suitable scheduling algorithms available for the resolution of each particular problem.

In order to execute the scheduling process it is necessary to clearly specify the problem to be solved. As mentioned above, scheduling problems have a set of characteristics that must be clearly and unequivocally defined. These characteristics include a class of factors related with the production environment, i.e. system and machines, and other classes that allow defining the characteristics of the jobs and resources and the performance measure or evaluation criterion. The first group of characteristics is related with the environment where the production is carried out. The manufacturing environment specification is denoted by $\alpha$ and includes the production system type definition and, eventually, the indication of the number of machines that exist in that production system. Typical such systems include several types of flow shops usually used in Product Oriented Manufacturing Systems (POMS) (Silva, 2001) and in cellular manufacturing systems. Other characteristics associated with scheduling problems, important and necessary for an adequate characterization of problems, are the constrains imposed to the manufacturing environment and resources $(\beta)$, e.g. machines and operators, and job processing conditions. Some important processing conditions are, for example, related with the existence of auxiliary resources, like robots and transportation devices and/or the existence of buffers, among others factors. Table 1 shows partial information about the problem classification notation in the form of $\alpha|\beta| \gamma$ (Varela, 1999) that serves as a basis for the XML-based problem specification model underlying to this work. This notation is based on notations proposed by (Blazewics, 1996), (Brucker, 1995) and (Jordan, 1996), as well as on other information presented by (Morton, 1993) and by other authors (Pinedo, 1995), (Artiba, 1997), and (Chrétienne, 1995).

The classification nomenclature includes a wide range of factors, which may be combined in many ways. Some of those factors are related with constraints, such as precedence constraints. In presence of this kind of constraint and under forward scheduling, a job can be started only after all of its predecessor jobs have been completed. Another usual constraint is associated with processing times and affects job start and completion times.

The use of this notation can be illustrated by the example " $\mathrm{F} \mid \mathrm{Cmax}$ " which reads as: "Scheduling of non-preemptable and independent tasks of arbitrary processing times (lengths), arriving to the system at time 0 , on a (simple) flow shop, in order to minimize schedule length".

Table 1 - A sample of scheduling problems characteristics.

\begin{tabular}{|c|c|l|l|}
\hline Class & Factor & \multicolumn{1}{|c|}{ Description } & \multicolumn{1}{c|}{ Value } \\
\hline \multirow{3}{*}{$\alpha$} & $\alpha 1$ & manufacturing system type & $0, P, Q, R, F, \mathrm{~J}, \mathrm{O}, \mathrm{X}, \mathrm{PMPM}, \ldots$ \\
\cline { 2 - 4 } & $\alpha 2$ & Number of machines & $0, k$ \\
\hline \multirow{4}{*}{$\beta$} & $\beta 1$ & Job/operation preemption & 0, pmtn \\
\cline { 2 - 4 } & $\beta 2$ & Precedence constraints & prec, chain, tree, sp-graph, $\ldots$ \\
\cline { 2 - 4 } & $\beta 3$ & Ready times & $0, r_{\mathrm{j}}$ \\
\cline { 2 - 4 } & $\beta 4$ & Restrictions on processing times & $p_{\mathrm{i}}=1, p_{\mathrm{ij}}=1, p_{\mathrm{i}}=\mathrm{p}, \mathrm{p}_{\mathrm{in}} \leq p_{\mathrm{i}} \leq \mathrm{p}_{\mathrm{pup}}, \ldots$ \\
\hline & $\ldots$ & $\ldots$ & $\ldots$ \\
\hline$\gamma$ & $\gamma$ & Performance measure & $\mathrm{C}_{\max }, \Sigma \mathrm{C}_{\mathrm{j}}, \sum \mathrm{w}_{\mathrm{i}} \mathrm{C}_{\mathrm{j}}, \mathrm{L}_{\max }, \sum \mathrm{T}_{\mathrm{j}}, \ldots$ \\
\hline
\end{tabular}


Table 2 shows some typical examples of scheduling problems and algorithms, based on (Bruker, 1995). It is a small sample for makespan flow shop scheduling problems among a vast universe of possible ones. This information is stored in the system knowledge base in order to help the user to identify their problems. The system also helps the user to assign methods to problems. This is further explained in the next section.

Table 2 - A sample of scheduling algorithms attributed to problems.

\begin{tabular}{|l|l|l|}
\hline \multicolumn{1}{|c|}{ Problem classes } & \multicolumn{1}{|c|}{ Algorithm reference } & \multicolumn{1}{c|}{ Observations } \\
\hline F2 ||Cmax & Johnson (1954) & $\begin{array}{l}\text { Maximal polynomially solvable } \\
\text { Without preemption }\end{array}$ \\
\hline F2 $|\mathrm{rj}| \mathrm{Cmax}$ & Lenstra et al (1977) & $\begin{array}{l}\text { Minimal NP-hard } \\
\text { Without preemption }\end{array}$ \\
\hline F2 | rj; no-wait |Cmax & Roeck (1984) & $\begin{array}{l}\text { Maximal polynomially solvable } \\
\text { With no wait }\end{array}$ \\
\hline F3 | pmtn |Cmax & $\begin{array}{l}\text { Gonzalez \& Sahni (1978) } \\
\text { Cho \& Sahni (1981) }\end{array}$ & $\begin{array}{l}\text { Maximal polynomially solvable } \\
\text { With preemption }\end{array}$ \\
\hline F|pji=1; prec $\mid \mathrm{Cmax}$ & $\begin{array}{l}\text { Leung et al (1984) } \\
\text { Timkovsky (1998) }\end{array}$ & $\begin{array}{l}\text { Minimal NP-hard } \\
\text { Without preemption }\end{array}$ \\
\hline FMPT $|\mathrm{n}=3| \mathrm{Cmax}$ & Kraemer (1995) & $\begin{array}{l}\text { Minimal NP-hard } \\
\text { With multiprocessor task }\end{array}$ \\
\hline FMPM, 3 | Cmax & Garey et al (1976) & $\begin{array}{l}\text { Minimal NP-hard } \\
\text { With multipurpose machines }\end{array}$ \\
\hline FMPM, $\mathrm{m}|\mathrm{rj} ; \mathrm{pji}=1| \mathrm{Cmax}$ & Brucker et al (1997) & $\begin{array}{l}\text { Maximal polynomially solvable } \\
\text { With multipurpose machines }\end{array}$ \\
\hline
\end{tabular}

\section{SYSTEM FUNCTIONALITIES}

Since 1995 great happenings have changed the world of information technology, especially the emergence of new Internet technologies. The eXtensible Markup Language (XML) is one of those new technologies that has been having a wide acceptance and caused great impact on Internet real world applications, since its release by the World Wide Web Consortium (W3C) in 1998 (Ceponkus, 1999). XML enables to describe structures and meanings of data with a simple syntax and is an ideal candidate format for exchanging and processing data through the Internet. Other advantages of an XML based representation are its openness, simplicity and scalability (Abiteboul, 2000). This is one of the main reasons why we have chosen XML to develop our application. For details about XML and related technologies (DTD, XSL, XML Schemas, Namespaces, etc.) see, for example (Ceponkus, 1999), (Abiteboul, 2000) or (Harper, 2001).

Web applications can use XML for local data processing, for showing multiple views of the data and for representing more complex data structures. Therefore, XML may guarantee the future utilization of data formats and the exchange of data structures, so that the web documents and the platforms become more robust for systems integration (Aparício, 2001). XML based data exchange is becoming very popular in global manufacturing, and this will cause connectivity becoming more and more convenient and necessary.

Some XML applications, which are more or less related with this work, are PDML (Product Data Markup Language), RDF (Resource Description Format) and STEPml (Pardi, 1999). Other XML specifications devoted to manufacturing 
processes are JDF (Job Definition Format), PSL (Process Specification Language), PIX-ML (Product Information Exchange), PIF (Process Interchange Format) and XML-based workflow (Harper, 2001).

As it has been said before, the main purpose of this work consists on trying to improve the resolution of scheduling problems. The system we are developing has a main element that is an interface for introduction, validation, and transformation of manufacturing scheduling data. The interface is mainly controlled by DTD and XSL documents stored in a database on a server. The system allows the execution of either local or remote algorithms. On remote executions, XML data is easily read and processed in every computer system since it is stored and sent as strings.

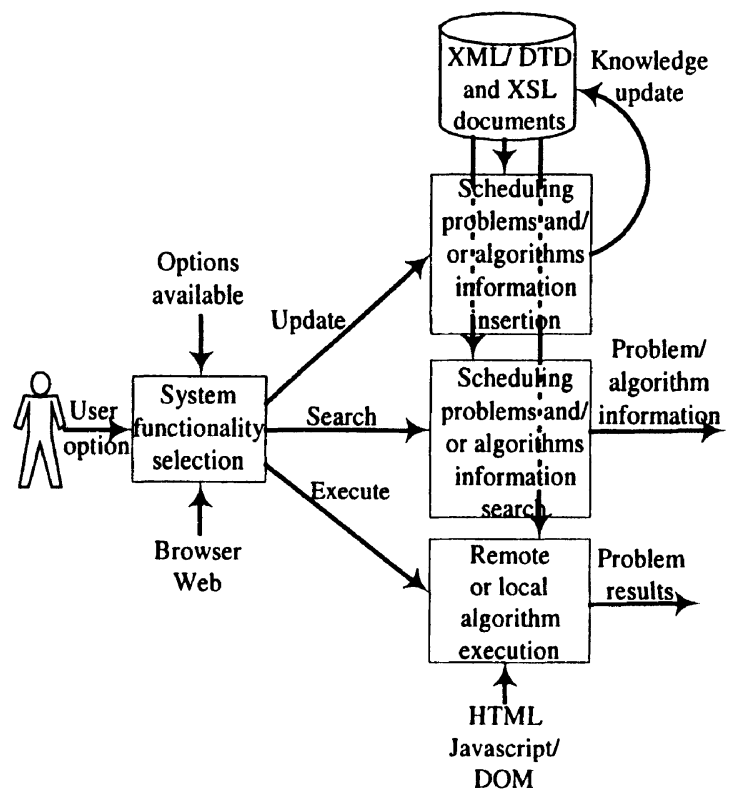

Figure 1- Web-system functionalities.

The fundamental system functionalities are those related to information modeling, which can be summarized as follows:

- Classification and identification of problems, by using the notation previously presented in section 2 .

- Classification and identification of scheduling methods and algorithms.

- Association of scheduling algorithms to scheduling problems.

- Ability to solve scheduling problems, through the selection of one or several algorithms, allowing comparison of results.

The scheduling information is stored in XML documents (e.g. problems.xml, algorithms.xml and implementations.xml, c.f. Figure 2). These documents are validated by corresponding Document Type Definition documents (DTDs), before being put in the XML database. Users can seek for scheduling problem classes, algorithms information and update, algorithms execution and scheduling problem results presentation and storage. The data can be shown in different views, using existing XSL documents, adequate for each specific visualization request.

Figure 2 illustrates the main update options available, underlying to the previously general update option shown in Figure 1. 


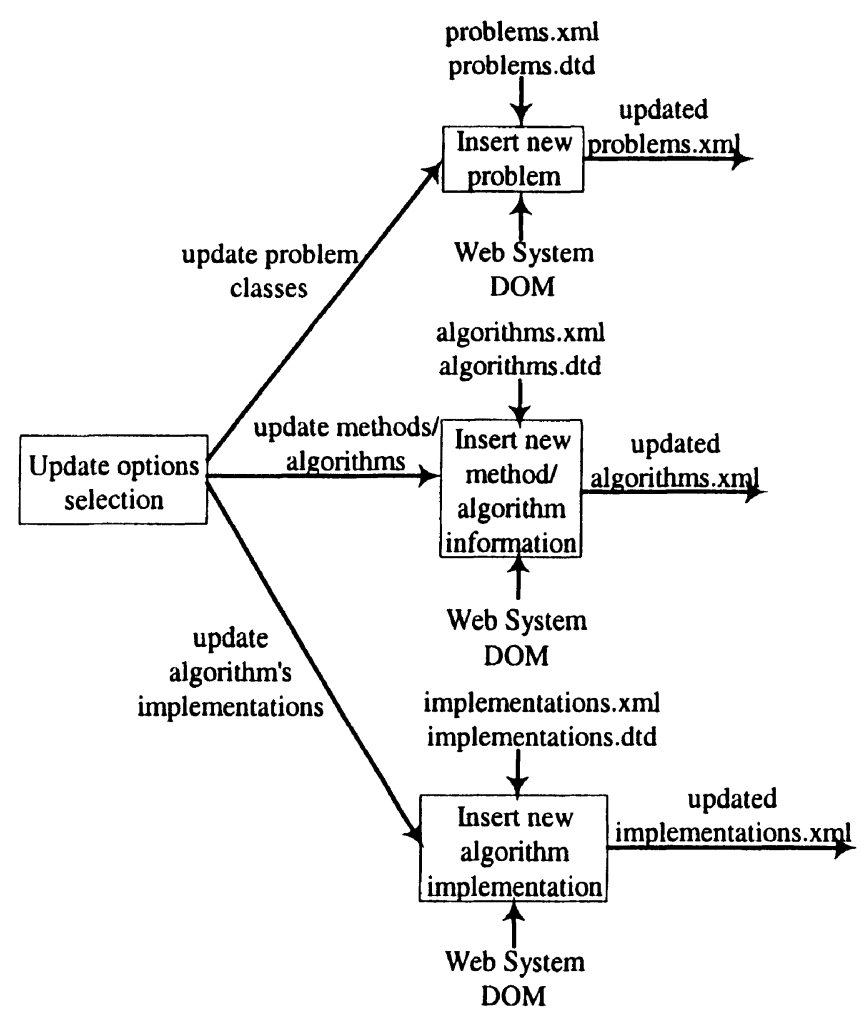

Figure 2 - System's update options.

\section{XML SPECIFICATION PRIMITIVES: AN EXAMPLE}

This section presents an XML-based specification of scheduling concepts. In a narrow sense, the specification of scheduling concepts gives us syntax of data description for this kind of scheduling problems, but in a broader sense, it can be seen as a general modeling form of such problems. The proposed specification framework provides a general model of scheduling problems and related concepts, and consequently a method of describing each particular problem and algorithm.

With respect to the performance measurement parameters, we think that objectives cannot be put together into one parameter. In other words, the XML specification provides a set of important parameters necessary for the most common kind of scheduling decisions. Then a planner chooses his/her preferences according to the circumstances. However, the XML specification allows users to add their own domain specific performance measures to be considered.

Primitive resource means workstations, machines, equipments, tools, labors, and so on. In these problems a job represents an action that has certain time duration. During that time, the job changes status of inventories of corresponding items, occupying or loading some particular resources. The jobs need some resources and produce some outputs. In XML specification, these objects are referred as items. From the viewpoint of Scheduling Problems, objects in the shop floor can be classified as resource category or as item category. If the target objects are produced or consumed by the job, they are defined as items. Otherwise, if they are renewable after the job being completed, we define them as resources. 
In defining job release orders, many attributes have to be considered such as item, quantity, location or destination, due date, processing time, and so on.

One of the most critical success factors for implementation of shop floor scheduling systems is the possibility of dealing with various constraints in each kind of production process, as explained in section 2 .

When the designer deals with XML, he/she writes tags hierarchy as elements, and possible attributes. These structures are concerned as a kind of syntax for each application domain. According to that syntax, a XML parser for the particular application translates data for each problem.

Let us now look at a concrete example. Suppose we want to introduce a new problem class. Figure 3 illustrates the system's interface that can be used for the definition of the problem main characteristics. It shows the input data for the problem class FMPM, m| rj, pji=1, n| Cmax, which reads: "Scheduling of nonpreemptable and independent tasks with unit processing time lengths, arriving to the system at time rj, on a multipurpose flow shop, with an arbitrary number of machines $\mathrm{m}$, in order to minimize schedule length".

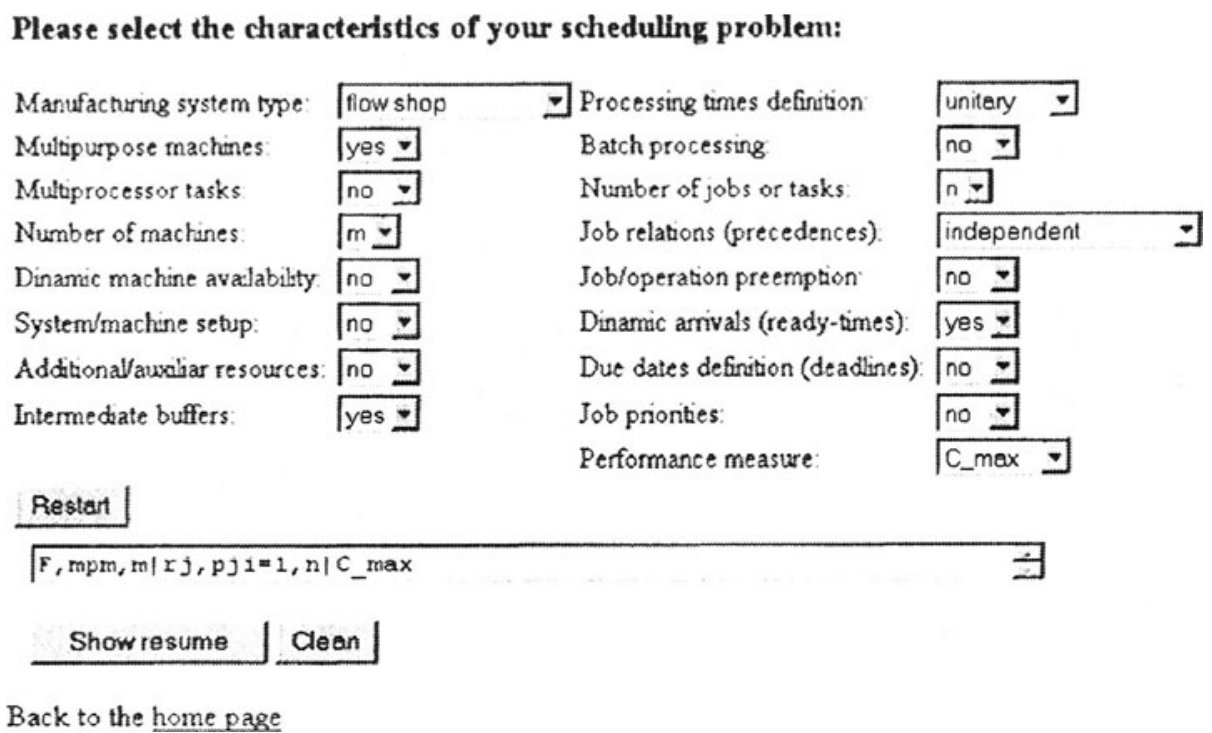

Figure 3 - A problem characterization.

This interface enables only valid data insertion, accordingly to a DTD (problems.dtd), which enables validating the user data input, allowing only valid data introduction according to the underlying scheduling problem characterization schema used, previously presented in section 2 (c.f. Table 1).

Listing 1 shows the DTD code, which is used for controlling the information related to problem classes identification and insertion in the correspondent XML document. 
Listing 1 - Problems.dtd.

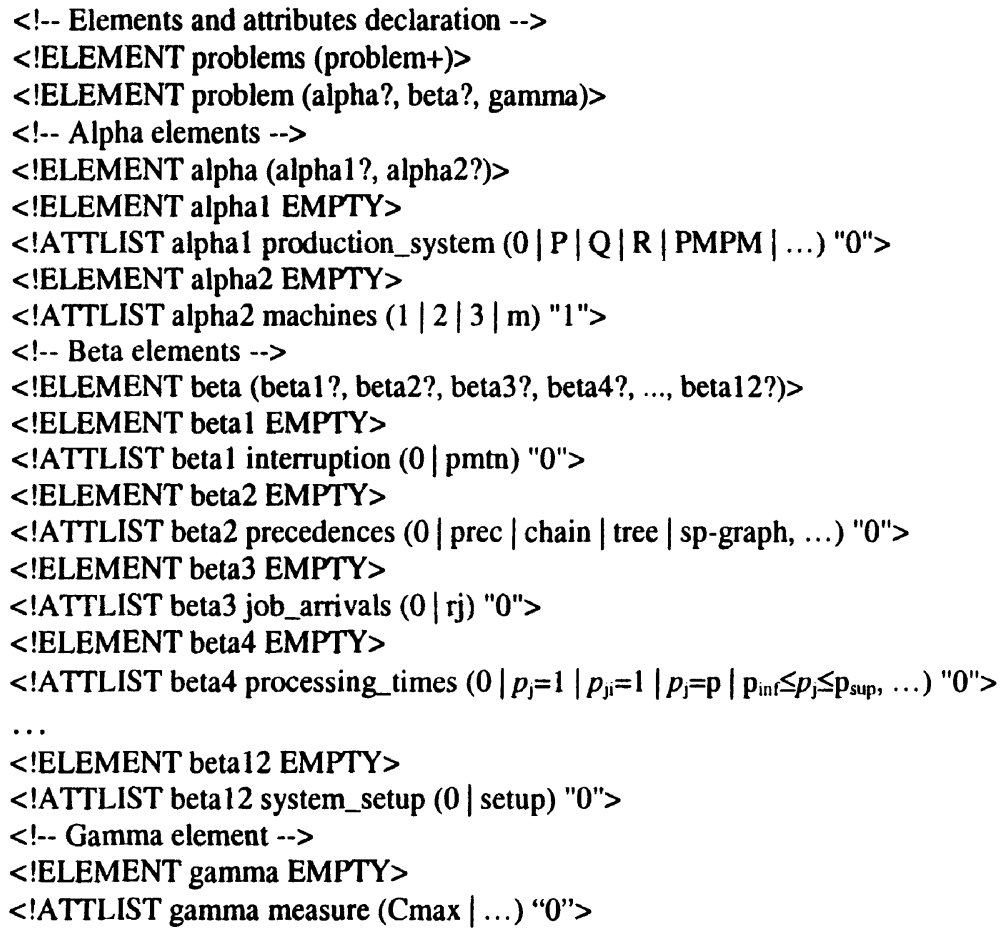

After having confirmed the problem class, submitted it, and validated it by the system, its information is then added to the correspondent XML document (updated problems.xml), as illustrated in section 3 (c.f. Figure 2). Listing 2 shows a sample of that document, which contains only the information associated to the problem class just added. In that specification the tag <problems $>$ is the root element and can have several $<$ problem $>$ tags as second level tags. These tags, on the other hand, include tags such as <alpha>, <beta> and <gamma> which, in turn, can be further subdivided in other third level tags.

\section{Listing 2 - Problems.xml.}

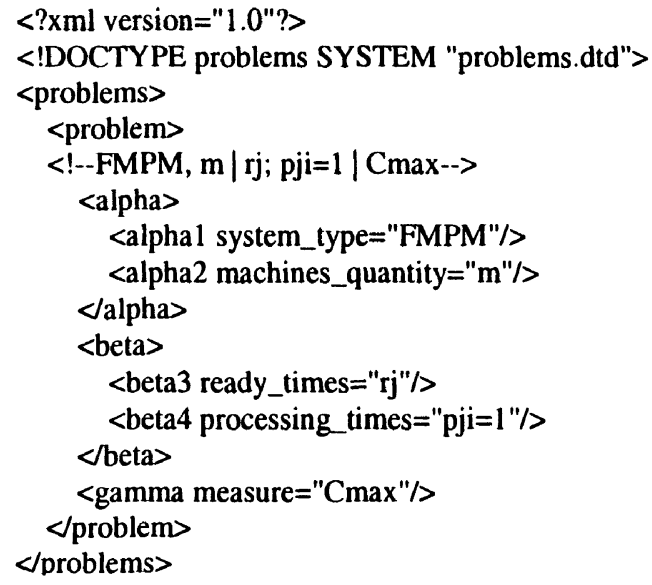


Listing 1 acts as a model for the description of problem according to Table 1, and Listing 2 acts as the actual repository of that information.

Furthermore, problems.dtd acts as a valuator for information being input to the knowledge base about problems (problems.xml).

For the algorithm execution, of local or remotely registered algorithms, it is still necessary to have DTDs, which describe and control their input and output data. In this case, we also need to have an interface that transforms the problem data, introduced by the user, through the problem identification process, into a valid input data format for the resolution algorithms and this is controlled through the appropriate DTDs. The same happens in the opposite direction, when we need to transform the algorithm results in valid problem results that can be properly processed: visualized and added to the corresponding XML documents.

It is still an open question how the Resource Description Format (Pardi, 1999) can be used for describing resources in our system. It seam that the RDF is too general for our purposes but an adequate subset of the vocabulary may be used in the system.

\section{CONCLUSIONS}

In manufacturing enterprises, it is important nowadays, as a competitive strategy, to explore and use software applications, now becoming available through the Internet and Intranets, for solving scheduling problems.

This communication proposes an XML-based specification framework for production scheduling concepts modeling, in a web-based production scheduling decision support system. Some of the important functions include the ability to represent scheduling problems and the identification and execution of appropriate available algorithms to solve them.

In order to make possible flexible communication among different scheduling applications, it is used an XML-based data modeling. This specification contributes to the improvement of the scheduling processes, by allowing an easy selection of several alternative available algorithms for problem solving, as well as an easy maintenance of the knowledge base itself. This primarily includes both scheduling problems and algorithms, which are available through the Internet. It is also an adequate specification format for the exchange of data, since it enables to handle with loosely coupled systems and with complex hierarchical data.

With the addition of DTDs, XML can handle much more complex tasks, but the DTDs also have some negative aspects, and their main disadvantages are related to the source code, which is not XML code. Therefore, in terms of future work we aim to transform them into XML Schemas, which are more easily integrated and used in the web system.

The XML based specification can be generated and visualized by computers in appropriate and different ways. An important issue is that the data representation model is general, accommodating a large variety of production scheduling problems. 


\section{REFERENCES}

1. Abiteboul S et al, Data on the web - from relations to semi structured data and XML. USA: Morgan Kaufmann Publishers, 2000.

2. Aparício JN et al, Applications development with XML. Portugal: New University of Lisbon, 2001.

3. Artiba A, Elmaghraby SE, The planning and scheduling of production systems. UK: Chapman \& Hall, 1997.

4. Blazewics J et al, Scheduling computer and manufacturing processes. Germany: Springer-Verlag, 1996.

5. Brucker P, Scheduling algorithms. Germany: Springer-Verlag, 1995.

6. Ceponkus A, Hoodbhoy F, Applied XML. USA: Wiley Computer Publishing, 1999.

7. Chrétienne P et al, Scheduling theory and its applications. England: John Wiley \& Sons Inc., 1995.

8. Harper F, XML standards and tools. USA: eXcelon Corporation, 2001.

9. Jordan C, Batching and scheduling. Germany: Springer-Verlag, 1996.

10. Morton T, Pentico DW, Heuristic scheduling systems. USA: John Wiley \& Sons Inc., 1993.

11. Pardi WJ, XML: Enabling next-generation web applications. USA: Microsoft Press, 1999.

12. Pinedo M, Scheduling theory, algorithms and systems. USA: Prentice-Hall Inc., 1995.

13. Silva SC, Alves AC, SPOP - Sistemas de Produção Orientados ao Produto, TeamWork' 2001 Conference, Lisbon, Conference, Lisbon, Portugal, 2001; 1-19.

14. Varela LR, Automatic scheduling algorithms selection. Portugal: Msc. Thesis, University of Minho, 1999. 